\title{
Esscher Transforms and Consumption-Based Models
}

\author{
Alex Badescu* \\ Department of Mathematics and Statistics \\ University of Calgary \\ Calgary, Alberta, \\ Canada
}

*This is joint work with Robert J. Elliott from University of Adelaide and University of Calgary, and Tak Kuen Siu from Macquarie University, Australia. 


\section{Outline of the presentation:}

- Introduction and literature review

- Conditional Esscher transform and equilibrium measures

- Conditional Esscher-Girsanov transforms

- Numerical results and conclusions 


\section{$\$ 1$. Introduction and literature review}

- Consider a discrete-time economy with time index set $\mathcal{T}=$ $\{t \mid t=0,1,2, \cdots, T\}$

- Let $M:=\left\{M_{t}\right\}_{t \in \mathcal{T}}$ be a non-negative, $\mathcal{F}$-adapted stochastic discount factor (SDF) process defined on $(\Omega, \mathcal{F}, \mathcal{P})$

- The price at time $t, \Pi_{t}$, of a payoff at $t+1, X_{t+1}$ :

$$
\Pi_{t}=E^{P}\left[M_{t+1} X_{t+1} \mid \mathcal{F}_{t}\right]
$$


- $c_{t}$ denotes consumption at $t$

- $u\left(c_{t}\right)$ is the utility function

- $\rho$ is the impatient factor of the economic agent per unit of time

- The first-order condition for an optimal consumption and portfolio choice:

$$
M_{t+1}=e^{-\rho} \frac{u^{\prime}\left(c_{t+1}\right)}{u^{\prime}\left(c_{t}\right)}
$$


- The log return process, $y_{t}$, under the physical measure $P$ :

$$
\begin{aligned}
y_{t} & =r_{t}+\lambda_{t} \sigma_{t}-\kappa_{\varepsilon_{t}}^{P}\left(\sigma_{t}\right)+\sigma_{t} \varepsilon_{t} \\
\varepsilon_{t} & \sim F(0,1) \\
\sigma_{t} & =a\left(\sigma_{t-1}, \sigma_{t-2}, \ldots \sigma_{1}, \varepsilon_{t-1}, \varepsilon_{t-2}, \ldots, \varepsilon_{1}\right) .
\end{aligned}
$$

- $\kappa_{\varepsilon_{t}}^{P}(z):=\ln M_{\varepsilon_{t}}^{P}(z)=\ln E^{P}\left[e^{z \varepsilon_{t}} \mid \mathcal{F}_{t-1}\right]<\infty, \quad|z|<h$

- If $Q$ is an equivalent martingale pricing measure, it can be related to the SDF by the following Radon-Nikodym derivative:

$$
\left.\frac{d Q}{d P}\right|_{\mathcal{F}_{T}}:=\exp \left(\sum_{t=1}^{T} r_{t}\right) \prod_{t=1}^{T} M_{t}
$$


- Rubinstein (1976) and Brennan (1979) - risk-neutral valuation relationship (RNVR) - joint normality of asset returns and marginal utility of consumption.

- Camara (1999) - extended the RNVR when the aggregate consumption and the random payoff of the underlying asset follow a bivariate three parameter lognormal distribution closed form solutions for European call options under this setting

- Camara (2003) - proposed a new set of equilibrium RNVRs - aggregate wealth and underlying asset have transformed normal distributions 


\section{Elliot and Madan (1998) - extended Girsanov principle}

- The Radon-Nikodym derivative:

$$
\left.\frac{d Q}{d P}\right|_{\mathcal{F}_{T}}:=\prod_{t=1}^{T} \frac{f_{\varepsilon_{t}}\left(\varepsilon_{t}+\lambda_{t}\right)}{f_{\varepsilon_{t}}\left(\varepsilon_{t}\right)}
$$

- $f_{\varepsilon_{t}}$ - the $\mathcal{F}_{t-1}$-conditional pdf of $y_{t}$ under $P$

- Consistent with hedging strategies that minimize the riskadjusted cost of hedging

- Schroder (2004) - relates this transformation to equilibrium pricing measures for some special cases of distributions of the return process 
§3. Conditional Esscher transform and equilibrium measures

- Definition 1 Let $\theta_{t}$ be an $\mathcal{F}_{t}$ predictable process, for each $t \in \mathcal{T} \backslash\{0\})$. The probability measure $Q^{\text {ess }}$ defined by:

$$
\left.\frac{d Q^{e s s}}{d P}\right|_{\mathcal{F}_{T}}=\prod_{t=1}^{T} \frac{e^{\theta_{t} y_{t}}}{M_{y_{t} \mid \mathcal{F}_{t-1}}^{P}\left(\theta_{t}\right)}
$$

is called the conditional Esscher transformed measure of $P$, generated by the return processes $y$ and the family $\theta$ of Esscher parameters, with respect to the filtration $F$.

- the Esscher parameter $\theta_{t}$ is the solution of the following equation:

$$
M_{y_{t} \mid \mathcal{F}_{t-1}}^{P}\left(1+\theta_{t}\right)=e^{r_{t}} M_{y_{t} \mid \mathcal{F}_{t-1}}^{P}\left(\theta_{t}\right)
$$


Proposition 1 Suppose the following conditions hold:

(a) The utility function $u$ is of exponential form:

$$
u\left(c_{t}\right)=\frac{1-e^{-R c_{t}}}{R}
$$

(b) for any $t \in \mathcal{T}$, the change in aggregate consumption satisfies the following, time-series, regression model under $P$ :

$$
\Delta c_{t}=\alpha_{t}+\beta_{t} y_{t}+\gamma_{t} \eta_{t}, \quad t \in \mathcal{T} \backslash\{0\}
$$

where $\eta_{t} \sim F(0,1)$ iid and independent of $y_{t}$

Then an equivalent martingale measure based on the equilibrium SDF coincides with that from the conditional Esscher transform. 
- $\theta_{t}=-R \beta_{t}, \quad t \in \mathcal{T} \backslash\{0\}$

- The Esscher parameter is proportional to the "beta" risk in the changes of aggregate consumption explained by the return of the risky asset.

- $R$ is the coefficient of absolute risk aversion

- The market price of risk:

$$
\lambda_{t}=\frac{\kappa_{\varepsilon_{t}}^{P}\left(\sigma_{t}\right)+\kappa_{\varepsilon_{t}}^{P}\left(-R \beta_{t} \sigma_{t}\right)-\kappa_{\varepsilon_{t}}^{P}\left(\left(1-R \beta_{t}\right) \sigma_{t}\right)}{\sigma_{t}}
$$


Proposition 2 Suppose the following conditions hold:

(a) The utility function $u$ is of isoelastic form:

$$
u\left(c_{t}\right)=\frac{c_{t}^{1-R}-1}{1-R}
$$

(b) for any $t \in \mathcal{T}$, the change in logarithm aggregate consumption satisfies the following, time-series, regression model under P:

$$
\Delta \mathrm{ln} c_{t}=\alpha_{t}+\beta_{t} y_{t}+\gamma_{t} \eta_{t}, \quad t \in \mathcal{T} \backslash\{0\} .
$$

where $\eta_{t} \sim F(0,1)$ iid and independent of $y_{t}$

Then an equivalent martingale measure based on the equilibrium SDF coincides with that from the conditional Esscher transform. 
- Consistency with consumption CAPM based on recursive utility - Epstein and Zin $(1989,1991)$

- The level of utility at any time point $t$ be:

$$
U_{t}=W\left(c_{t}, E\left[U_{t+1}^{\varrho} \mid \mathcal{F}_{t}\right]^{1 / \varrho}\right), \quad 0 \neq \varrho<1 .
$$

- $\varrho$ is the risk aversion parameter and the degree of risk aversion increases as $\varrho$ does; $W$ is the "aggregator" function having the following form:

$$
W(c, z)=\left[\left(1-e^{-\rho}\right) c^{\phi}+e^{-\rho} z^{\phi}\right]^{1 / \phi}, \quad 0 \neq \phi<1
$$

- $c$ and $z$ are both positive; $\phi$ is a parameter which reflects the inter-temporal substitution; $\rho$ is the impatience factor 
- $\varrho=1-R$, where $R$ is the constant coefficient of relative risk aversion

- The maximization problem leads to:

$$
E^{P}\left[e^{-\rho \varsigma}\left(\frac{c_{t}}{c_{t-1}}\right)^{\varsigma(\phi-1)}\left(\frac{S_{m t}}{S_{m t-1}}\right)^{\varsigma-1} \frac{S_{t}}{S_{t-1}} \mid \mathcal{F}_{t-1}\right]=1, \quad \forall t \in \mathcal{T} .
$$

where $S_{m t}$ is the price index of the market portfolio at time $t$ and $\varsigma=\varrho / \phi$

- The equilibrium SDF:

$$
M_{t}=e^{-\rho \varsigma}\left(\frac{c_{t}}{c_{t-1}}\right)^{\varsigma(\phi-1)}\left(\frac{S_{m t}}{S_{m t-1}}\right)^{\varsigma-1}, \quad \forall t \in \mathcal{T} .
$$


Proposition 3 For any $t \in \mathcal{T}$, we assume that the changes in the logarithm of the aggregate consumption and the return on the market portfolio, $y_{m t}:=\ln \left(S_{m t} / S_{m t-1}\right)$, satisfy the following regression model under $P$ :

$$
\left(\Delta \ln c_{t}, y_{m t}\right)^{T}=\underline{\alpha}_{t}+\underline{\beta}_{t} y_{t}+\operatorname{diag}\left(\underline{\eta_{t}}\right) \underline{\gamma}_{t} .
$$

Here $\underline{\alpha}_{t}:=\left(\alpha_{1 t}, \alpha_{2 t}\right)^{T}, \underline{\beta}_{t}:=\left(\beta_{1 t}, \beta_{2 t}\right)^{T}, \underline{\gamma}_{t}:=\left(\gamma_{1 t}, \gamma_{2 t}\right)^{T}$ are 2-dimensional $\mathcal{F}$-predictable processes and $\underline{\eta}_{t}:=\left(\eta_{1 t}, \eta_{2 t}\right)^{T}$ is a 2-dimensional vector of i.i.d. random variables with $\eta_{1 t}, \eta_{2 t} \sim$ $F(0,1)$ independent of $y_{t}$ for any $t \in \mathcal{T}$ and also independent of each other. Here diag $\left(\eta_{t}\right)$ is the diagonal matrix formed with the elements of $\eta_{t}$.

Then an equivalent martingale measure based on the above equilibrium SDF is consistent with that from the conditional Esscher transform. 
- The Esscher parameter is given by:

$$
\theta_{t}=\varsigma(\phi-1) \beta_{1 t}+(\varsigma-1) \beta_{2 t} .
$$

- $\theta_{t}$ - weighted average between a fraction of the "beta" risk in the changes of logarithm aggregate consumption explained by the return of the risky asset and the negative "beta" risk of the return on the market portfolio explained by the return on the risky asset

- If $\varsigma=1$, then $\theta_{t}=-R \operatorname{cov}\left(\Delta \log c_{t}, y_{t}\right) / \sigma_{t}^{2}$ - same as standard CAPM

- If $\varsigma=0$, then $\left.\theta_{t}=\operatorname{cov}\left(y_{m t}, y_{t}\right)\right) / \sigma_{t}^{2}$ 
§4. Conditional Esscher-Girsanov transform and its generalization

- The conditional Esscher-Girsanov transform was introduced by Goovaerts and Laeven (2008)

- Define the function $\Psi\left(\varepsilon_{t}\right)$ :

$$
\Psi\left(\varepsilon_{t}\right)=\Phi^{-1}\left(F\left(\varepsilon_{t}\right)\right)
$$

- $\Phi$ is the cdf of a standard normal random variable

- $\Psi\left(\varepsilon_{t}\right)$ is a standard normal random variable under $P$ conditional on $\mathcal{F}_{t-1}$. 
Definition 2 (Conditional Esscher-Girsanov transform) Let $\delta:=$ $\left\{\delta_{t}\right\}_{t \in \mathcal{T}}$ be an F-predictable process and let $h$ be a positive constant. The probability measure $Q^{e s s g}$ defined by:

$$
\left.\frac{d Q^{e s s g}}{d P}\right|_{\mathcal{F}_{T}}=\prod_{t=1}^{T} e^{h \delta_{t} \sigma_{t} \Psi\left(\varepsilon_{t}\right)-\frac{1}{2} h^{2} \delta_{t}^{2} \sigma_{t}^{2}}
$$

is called the conditional Esscher-Girsanov transformed measure of $P$ with respect to the filtration $\mathcal{F}_{t}$ for parameter $h$ and penalty process $\delta$.

1. The reason of having 2 parameters is to separate the risk aversion parameter from the risk premium

2. If $\mathrm{h}=1$, the Radon-Nikodym derivative resembles a conditional version of Wang transform. 
Proposition 4 Under $Q^{e s s g}$ the risk-neutralized dynamics of the asset return process are given by:

$y_{t}=m_{t}+\sigma_{t} \Psi^{-1}\left(\xi_{t}+h \delta_{t} \sigma_{t}\right), \quad \xi_{t} \sim N(0,1)$

$\sigma_{t}=a\left(\sigma_{t-1}, \sigma_{t-2}, \ldots, \Psi^{-1}\left(\xi_{t-1}+h \delta_{t-1} \sigma_{t-1}\right), \Psi^{-1}\left(\xi_{t-2}+h \delta_{t-2} \sigma_{t-2}\right), \ldots\right)$

where $\xi_{t}$ is a sequence of i.i.d. standard Gaussian random variables under $Q^{e s s g}$, and $h$ and $\delta_{t}$ satisfy:

$$
M_{\left(\varepsilon_{t}, \Psi\left(\varepsilon_{t}\right)\right) \mid \mathcal{F}_{t-1}}^{P}\left(\sigma_{t}, h \delta_{t} \sigma_{t}\right)=e^{r_{t}-m_{t}+\frac{1}{2} h^{2} \delta_{t}^{2} \sigma_{t}^{2}}
$$

Note: This is similar to the risk neutralized dynamic obtained by Duan (1999) using his generalized local RNVR 
Proposition 5 Let $\equiv\left(c_{t}\right)$ be the inverse Gaussian transformation of $c_{t}$ through its conditional c.d.f. (ie. $\equiv\left(c_{t}\right)$ is standard normally distributed conditional on $\left.\mathcal{F}_{t-1}\right)$. If $\Psi\left(\varepsilon_{t}\right)$ and $\Delta \equiv\left(c_{t}\right)$ have a conditionally bivariate normal distribution with respect to $\mathcal{F}_{t-1}$ under $P$ and the marginal utility function is of the form:

$$
u^{\prime}\left(c_{t}\right)=e^{-R \equiv\left(c_{t}\right)}, \quad R>0
$$

then the risk neutral measure constructed based on the standard equilibrium SDF is consistent with the conditional EsscherGirsanov transformation.

1. $R$ is no longer the Arrow-Pratt measure of absolute risk aversion

2. It follows that $h=-R$ and $\delta_{t}=\operatorname{cov}\left(\Delta \equiv\left(c_{t}\right), \Psi\left(\varepsilon_{t}\right) \mid \mathcal{F}_{t-1}\right) / \sigma_{t}$ 
- If we assume $m_{t}=r_{t}+\lambda_{t} \sigma_{t}-\kappa_{\varepsilon_{t}}^{P}\left(\sigma_{t}\right)$, then the market price of risk:

$$
\lambda_{t}=\frac{\kappa_{\varepsilon_{t}}^{P}\left(\sigma_{t}\right)+\frac{1}{2} R^{2} \delta_{t}^{2} \sigma_{t}^{2}-\kappa_{\left(\varepsilon_{t}, \Psi\left(\varepsilon_{t}\right)\right) \mid \mathcal{F}_{t-1}}^{P}\left(\sigma_{t},-R \delta_{t} \sigma_{t}\right)}{\sigma_{t}} .
$$

- The unit risk premium is decomposed into the sum of a risk free component and a term which contains the risk preference parameter

- If the innovations are normally distributed, then $\lambda_{t}=R \delta_{t} \sigma_{t}$ same result as Esscher transform

- The choice of the inverse Gaussian transform can be extended to other distortion functions 
- Let $G$ be the cdf of a standardized random variable having a finite mgf

- Define the following transformation:

$$
\psi^{(G)}\left(\varepsilon_{t}\right)=G^{-1}\left(F\left(\varepsilon_{t}\right)\right)
$$

- $\Psi^{(G)}\left(\varepsilon_{t}\right)$ has an $\mathcal{F}_{t}$-conditionally standard $G$ distributed under $P$

- When $G=\Phi$ - Esscher-Girsanov transform 


\section{Definition 3 (Generalized Conditional Esscher-Girsanov transforn} Let $\delta^{(G)}:=\left\{\delta_{t}^{(G)}\right\}_{t \in \mathcal{T}}$ be an F-predictable process and let $h$ be a positive constant. The family of probability measures indexed by $G, Q^{(G)}$, defined by:

$$
\left.\frac{d Q^{(G)}}{d P}\right|_{\mathcal{F}_{T}}=\prod_{t=1}^{T} \frac{e^{h \delta_{t}^{(G)} \sigma_{t} \Psi^{(G)}\left(\varepsilon_{t}\right)}}{M_{\Psi(G)\left(\varepsilon_{t}\right) \mid \mathcal{F}_{t-1}}^{P}\left(h \delta_{t}^{(G)} \sigma_{t}\right)}
$$

is called the generalized conditional Esscher-Girsanov transformed measure of $P$ with respect to the filtration $\mathcal{F}_{t}$ with parameter $h$ and penalty process $\delta_{t}^{(G)}$.

Note: The parameters $h$ and $\delta_{t}^{(G)}$ satisfy the martingale equation:

$$
\left.M_{\left(\varepsilon_{t}, \Psi(G)\right.}^{P}\left(\varepsilon_{t}\right)\right)\left|\mathcal{F}_{t-1}\left(\sigma_{t}, h \delta_{t}^{(G)} \sigma_{t}\right)=e^{r_{t}-m_{t}} M_{\Psi(G)}^{P}\left(\varepsilon_{t}\right)\right| \mathcal{F}_{t-1}\left(h \delta_{t}^{(G)} \sigma_{t}\right)
$$


Proposition 6 Let $\equiv^{(G)}\left(c_{t}\right)$ be the $G$-transformation of $c_{t}$ through its conditional cdf (i.e. $\Xi^{(G)}\left(c_{t}\right)$ is standard $G$-distributed conditional on $\left.\mathcal{F}_{t-1}\right)$. If there exist three $\mathcal{F}_{t}$-predictable processes, $\alpha_{t}^{(G)}, \beta_{t}^{(G)}$, and $\gamma_{t}^{(G)}$ and a sequence of random variables $\eta_{t}$ independent of $\varepsilon_{t}$ such that $\eta_{t} \sim G(0,1)$ and:

$$
\Delta \Xi^{(G)}\left(c_{t}\right)=\alpha_{t}^{(G)}+\beta_{t}^{(G)} \psi^{(G)}\left(\varepsilon_{t}\right)+\gamma_{t}^{(G)} \eta_{t}
$$

then the risk neutral measure constructed based on the standard equilibrium SDF with marginal utility function satisfying:

$$
u^{\prime}\left(c_{t}\right)=e^{-R \equiv{ }^{(G)}\left(c_{t}\right)}, \quad R>0
$$

is consistent with the generalized conditional Esscher-Girsanov transform.

Note: The transformation parameters are $h=-R$ and $\delta_{t}^{(G)}=$ $\beta_{t}^{(G)} / \sigma_{t}=\operatorname{cov}\left(\Delta \Xi^{(G)}\left(c_{t}\right), \Psi^{(G)}\left(y_{t}\right) \mid \mathcal{F}_{t-1}\right) / \sigma_{t}$ 


\section{§5. Numerical results and conclusions}

- The return dynamic under $P$ :

$$
\begin{aligned}
y_{t} & =r+\lambda \sigma_{t}+\sigma_{t} \varepsilon_{t} \\
\varepsilon_{t} & \sim z(\alpha, \beta, \delta, \mu) \\
\sigma_{t}^{2} & =a_{0}+a_{1} \sigma_{t-1}^{2} \varepsilon_{t-1}^{2}+\gamma \mathrm{I}\left(\varepsilon_{t-1}<0\right) \sigma_{t-1}^{2} \varepsilon_{t-1}^{2}+b_{1} \sigma_{t-1}^{2}
\end{aligned}
$$

- the return pdf and cumulant generating functions:

$$
\begin{aligned}
f_{\varepsilon_{t}}(x, \alpha, \beta, \delta, \mu) & =\frac{1}{\delta B(\alpha, \beta)} \cdot \frac{(\exp [(x-\mu) / \delta])^{\alpha}}{(1+\exp [(x-\mu) / \delta])^{\alpha+\beta}} \\
\kappa_{\varepsilon_{t}}(u) & =\log \frac{B(\alpha+\delta u, \beta-\delta u)}{B(\alpha, \beta)}+\mu u
\end{aligned}
$$


- S\&P 500 returns data from January 3rd, 1988 to January 6th, 2004

- Estimate the model parameters by MLE

- Use Monte Carlo simulation (50,000 paths) to compute option prices for MCMM, Esscher transform and Esscher-Girsanov $(\mathrm{h}=1)$ transform

- Call options (STRICKNET INC) sampled every Wednesday at closing prices from January 7th, 2004 to December 29th, 2004. 
- 1582 European call options, average bid-ask spread is $\$ 1.13$ and average Call price is $\$ 18.36$.

- Model performance is tested using root mean squared error (RMSE):

$$
R M S E=\sqrt{\frac{1}{N} \sum_{i=1}^{N}\left(C_{i}^{\text {market }}-\hat{C}_{i}^{\text {model }}\right)^{2}}
$$




\section{Number of Call option contracts}

\begin{tabular}{c|c|c|c|c|c} 
Mo & $D T M<40$ & $40<D T M<80$ & $80<D T M<180$ & $D T M>180$ & All \\
\hline$[0.8,0.9)$ & 8 & 1 & 3 & 2 & 14 \\
{$[0.9,0.95)$} & 31 & 5 & 3 & 4 & 43 \\
{$[0.95,0.975)$} & 39 & 6 & 2 & 2 & 49 \\
{$[0.975,0.99)$} & 88 & 19 & 8 & 9 & 124 \\
{$[0.99,1.01)$} & 259 & 76 & 37 & 21 & 393 \\
{$[1.01,1.025)$} & 201 & 38 & 17 & 6 & 262 \\
{$[1.025,1.05)$} & 232 & 57 & 32 & 4 & 325 \\
{$[1.05,1.1)$} & 166 & 83 & 50 & 24 & 323 \\
{$[1.1,1.2]$} & 9 & 7 & 23 & 10 & 49 \\
\hline All & 1,033 & 292 & 175 & 82 & 1,582
\end{tabular}




\section{Average Call option prices}

\begin{tabular}{c|c|c|c|c|c} 
Mo & $D T M<40$ & $40<D T M<80$ & $80<D T M<180$ & $D T M>180$ & All \\
\hline$[0.8,0.9)$ & 165.00 & 177.80 & 173.93 & 152.65 & 166.06 \\
{$[0.9,0.95)$} & 82.65 & 88.22 & 106.30 & 119.53 & 88.38 \\
{$[0.95,0.975)$} & 46.54 & 52.75 & 67.40 & 92.85 & 50.04 \\
{$[0.975,0.99)$} & 25.08 & 40.99 & 61.25 & 75.06 & 33.48 \\
{$[0.99,1.01)$} & 13.47 & 30.88 & 47.69 & 65.37 & 22.83 \\
{$[1.01,1.025)$} & 5.59 & 19.80 & 38.21 & 55.50 & 10.91 \\
{$[1.025,1.05)$} & 2.72 & 11.82 & 28.79 & 47.33 & 7.43 \\
{$[1.05,1.1)$} & 0.67 & 4.27 & 14.93 & 26.54 & 5.73 \\
{$[1.1,1.2]$} & 0.13 & 0.37 & 4.62 & 11.05 & 4.50 \\
\hline All & 12.84 & 20.01 & 32.31 & 52.28 & 18.36
\end{tabular}




\section{Panel A. RMSE for $z$-TGARCH with MCMM}

\begin{tabular}{c|c|c|c|c|c} 
Mo & $D T M<40$ & $40<D T M<80$ & $80<D T M<180$ & $D T M>180$ & All \\
\hline$[0.8,0.9)$ & 1.11 & 1.53 & 4.83 & 2.27 & 2.57 \\
{$[0.9,0.95)$} & 1.53 & 2.63 & 4.44 & 3.86 & 2.29 \\
{$[0.95,0.975)$} & 2.34 & 4.11 & 6.27 & 1.69 & 2.86 \\
{$[0.975,0.99)$} & 1.80 & 4.50 & 5.03 & 7.07 & 3.27 \\
{$[0.99,1.01)$} & 1.98 & 3.81 & 5.48 & 6.69 & 3.26 \\
{$[1.01,1.025)$} & 1.47 & 3.65 & 5.29 & 5.94 & 2.49 \\
{$[1.025,1.05)$} & 1.03 & 2.48 & 5.13 & 5.21 & 2.18 \\
{$[1.05,1.1)$} & 0.52 & 1.37 & 2.92 & 4.07 & 1.78 \\
{$[1.1,1.2]$} & 0.12 & 0.17 & 0.83 & 2.12 & 1.12 \\
\hline All & 1.50 & 3.00 & 4.33 & 5.21 & 2.57
\end{tabular}


Panel B. RMSE for $z$-TGARCH with Esscher-Girsanov $(h=1)$ transform

\begin{tabular}{c|c|c|c|c|c} 
Mo & $D T M<40$ & $40<D T M<80$ & $80<D T M<180$ & $D T M>180$ & All \\
\hline$[0.8,0.9)$ & 1.13 & 2.39 & 3.93 & 1.43 & 2.18 \\
{$[0.9,0.95)$} & 1.52 & 2.37 & 3.94 & 3.09 & 2.07 \\
{$[0.95,0.975)$} & 2.29 & 3.74 & 5.37 & 1.74 & 2.68 \\
{$[0.975,0.99)$} & 1.72 & 4.42 & 4.64 & 6.28 & 3.06 \\
{$[0.99,1.01)$} & 1.93 & 3.57 & 4.80 & 5.75 & 2.97 \\
{$[1.01,1.025)$} & 1.45 & 3.46 & 4.73 & 5.22 & 2.33 \\
{$[1.025,1.05)$} & 1.02 & 2.30 & 4.53 & 5.01 & 1.98 \\
{$[1.05,1.1)$} & 0.51 & 1.26 & 2.55 & 3.28 & 1.53 \\
{$[1.1,1.2]$} & 0.12 & 0.17 & 0.60 & 1.79 & 0.91 \\
\hline All & 1.46 & 2.83 & 3.82 & 4.48 & 2.35
\end{tabular}




\section{Panel C. RMSE for z-TGARCH with Esscher transform}

\begin{tabular}{c|c|c|c|c|c} 
Mo & $D T M<40$ & $40<D T M<80$ & $80<D T M<180$ & $D T M>180$ & All \\
\hline$[0.8,0.9)$ & 0.98 & 1.11 & 3.97 & 1.99 & 2.14 \\
{$[0.9,0.95)$} & 1.50 & 2.60 & 3.79 & 2.93 & 2.05 \\
{$[0.95,0.975)$} & 2.22 & 3.77 & 4.70 & 2.12 & 2.60 \\
{$[0.975,0.99)$} & 1.70 & 4.18 & 4.09 & 5.74 & 2.86 \\
{$[0.99,1.01)$} & 1.89 & 3.44 & 4.49 & 5.24 & 2.83 \\
{$[1.01,1.025)$} & 1.42 & 3.40 & 4.38 & 4.38 & 2.22 \\
{$[1.025,1.05)$} & 1.01 & 2.27 & 4.37 & 4.11 & 1.93 \\
{$[1.05,1.1)$} & 0.51 & 1.27 & 2.33 & 3.06 & 1.44 \\
{$[1.1,1.2]$} & 0.12 & 0.16 & 0.61 & 1.66 & 0.86 \\
\hline All & 1.44 & 2.76 & 3.57 & 4.07 & 2.24
\end{tabular}


- Shows a consistency between Esscher-type transforms and stochastic discount factors constructed under equilibrium conditions in a general discrete time framework

- For conditional Esscher transform we identified risk neutral measures based on the standard Euler equation and recursive utility

- We derive new equilibrium SDFs consistent with the EsscherGirsanov principle and its generalization 


\section{$\sim$ Thank you !}




\section{References}

- Badescu, A.M., and Kulperger, R.J. (2008). GARCH option pricing: a semiparametric approach. Insurance: Mathematics and Economics, 43(1), 69-84.

- Badescu, A.M., Kulperger, R.J., and Lazar, E. (2008). GARCH option pricing: a semiparametric approach, to appear in Studies in Nonlinear Dynamics \& Econometrics, 12(2), Article 5.

- Bertholon Bertholon, H., Monfort, A., and Pegoraro, F. (2008). Econometric asset pricing modelling. Journal of Financial Econometrics, 6(4), 407-458. 
- Black, F., and Scholes, M. (1973). The pricing of options and corporate liabilities. Journal of Political Economy, 81, 637-659.

- Brennan, M.J. (1979). The pricing of contingent claims in discrete time models. Journal of Finance, 24(1), 53-68.

- Buhlmann, H. (1980). An economic premium principle. ASTIN Bulletin, 11(1), 52-60.

- Buhlmann, H. (1984). The general economic premium principle. ASTIN Bulletin, 14(1), 13-21. 
- Buhlmann, H., Delbaen, F., Embrechts, P. and Shiryaev, A.N. (1998). On Esscher transforms in discrete finance models. ASTIN Bulletin, 28(2), 171-186.

- Camara, A. (1999). An extended set of risk neutral valuation relationships for the pricing of contingent claims. Review of Derivative Research, 3, 67-83.

- Camara, A. (2003). A generalization of the Brennan-Rubinstein approach for the pricing derivatives. Journal of Finance, 2, 805-819.

- Christoffersen, P., Redouane E., Feunou, B., and Jacobs, K. (2008) Option Valuation with Conditional Heteroskedasticity and Non-Normality. working paper, McGill University. 
- Duan, J.-C. (1995). The GARCH option pricing model. Mathematical Finance, 5, 13-32.

- Duan, J.-C. (1999). Conditionally fat-tailed distributions and the volatility smile in options, unpublished manuscript, University of Toronto.

- Elliott, R.J., and Madan, D.B. (1998). A discrete time equivalent martingale measure. Mathematical Finance, 9(6), 825841.

- Epstein, L., and Zin, S. (1989). Substitution, risk aversion, and temporal behavior of consumption and asset returns: A theoretical framework. Econometrica, 57, 937-969. 
- Epstein, L., and Zin, S. (1991). Substitution, risk aversion, and temporal behavior of consumption and asset returns: An empirical analysis. Journal of Political Economy, 99, 263286.

- Gerber, H.U., and Shiu, E.S.W. (1994). Option pricing by Esscher transforms (with disscusions). Transactions of the Society of Actuaries 46, 99-191.

- Goovaerts, M., Laeven, R. (2008). Actuarial risk measures for financial derivative pricing. Insurance: Mathematics and Economics, 42(2), 540-547. 
- Iwaki, H., Kijima, M., and Morimotoc, Y. (2001). An economic premium principle in a multiperiod economy. Insurance: Mathematics and Economics, 28(3), 325-339.

- Lucas, R. (1978). Asset Pricing in an Exchange Economy. Econometrica, 46, 1429-1445.

- Rubinstein, M. (1976). The valuation of uncertain income streams and the pricing of options, Bell Journal of ECOnomics, 7, 407-425

- Siu, T.K., Tong, H., and Yang, H. (2004). On pricing derivatives under GARCH models: a dynamic Gerber-Shiu approach. North American Actuarial Journal, 8(3), 17-32. 
- Siu, T.K. (2009). Esscher Transform: From Actuarial Science to Quantitative Finance. Encyclopedia of Quantitative Finance. Forthcoming.

- Schroder, M. (2004). Risk-neutral parameter shifts and derivatives pricing in discrete time. Journal of Finance, 59(5), 2375-2402.

- Wang, S.S. (2000). A class of distortion operators for pricing financial and insurance risks. Journal of Risk and Insurance, $67(1), 15-36$.

- Wang, S.S. (2003). Equilibrium pricing transforms: New results on Bühlmann's 1980 economic model. ASTIN Bulletin, $32,213-234$. 